\title{
A phase II study of concurrent chemoradiotherapy and erlotinib for inoperable esophageal squamous cell carcinoma
}

\author{
Chuanhua Zhao ${ }^{1}$, Li Lin ${ }^{1}$, Jianzhi Liu ${ }^{1}$, Rongrui Liu ${ }^{1}$, Yuling Chen ${ }^{1}$, Feijiao Ge ${ }^{1}$, Ru \\ $\mathrm{Jia}^{1}$, Yang Jin ${ }^{1}$, Yan Wang ${ }^{1}$ and Jianming $X \mathbf{u}^{1}$ \\ ${ }^{1}$ Department of GI Oncology, 307 Hospital of PLA, Academy of Military Medical Sciences, Beijing, China \\ Correspondence to: Jianming $\mathrm{XU}$, email: jmxu2003@163.com \\ Keywords: esophageal cancer, epidermal growth factor receptor, chemoradiotherapy, erlotinib, paclitaxel \\ Received: March 27, $2016 \quad$ Accepted: May 23, $2016 \quad$ Published: June 02, 2016
}

\section{ABSTRACT}

Cisplatin-based concurrent chemoradiotherapy for patients with unresectable, locally advanced esophageal squamous cell carcinoma (ESCC) is associated with significant toxicities that are often intolerable. Prognosis for this subgroup of patients remains poor, and new therapeutic approaches are urgently needed. We investigated the efficacy and safety of paclitaxel combined with erlotinib and concurrent radiotherapy in patients with inoperable ESCC. Erlotinib $(150 \mathrm{mg})$ was administered daily for 60 days beginning at the start of radiotherapy, and paclitaxel $\left(45 \mathrm{mg} / \mathrm{m}^{2}\right)$ was administered weekly along with intensity modulated conformal radiotherapy (60 Gy in 30 fractions). The median follow-up time was 21 months. The associations between EGFR and VEGF expression and treatment outcome were evaluated. Among the 21 patients treated, the overall response rate $(C R+P R)$ was $85.6 \%$. The median LPFS, PFS and OS were: 17.5, 14.3, and 22.9 months, respectively. Treatment-related grade 3 toxicities included esophagitis (two patients) and hypoleukemia (one patient). Grade 4 pulmonary toxicity was observed in one patient. Patients expressing EGFR had longer PFS, while those expressing VEGF or with a history of smoking had worse outcomes. Weekly paclitaxel combined with erlotinib and concurrent radiotherapy shows promise as an effective, tolerated regimen for patients with inoperable ESCC.

\section{INTRODUCTION}

Esophageal cancer is the eighth most common cancer worldwide and the fourth most common in China [1]. At the time of diagnosis, two-thirds of patients will have tumors that are considered inoperable due to comorbidities or tumor extension [2]. Concurrent chemoradiotherapy (CRT) based on cisplatin and fluorouracil (PF) is the standard treatment for patients with inoperable, locally advanced esophageal squamous cell carcinoma (ESCC) [3]. This therapeutic approach reduces mortality somewhat but at a cost of increased toxicity, and the 5-year overall survival (OS) rate remains less than 20\% [4]. Clearly, new therapeutic agents and treatment strategies are urgently needed for this subgroup of patients.

Paclitaxel is a widely used chemotherapeutic agent that has demonstrated promising antitumor activity in patients with esophageal cancer [5]. A study comparing weekly paclitaxel with a PF regimen for ESCC patients undergoing synchronous combined chemotherapy and radiotherapy (RT) reported similar immediate curative effects and 1,3, and 5-year survival rates in the two groups, though greater digestive toxicity was observed in the PF group [6]. In China, weekly paclitaxel combined with RT is considered a feasible regimen for ESCC patients.

Epidermal growth factor receptor (EGFR) is overexpressed in $30-70 \%$ of ESCC cases and is associated with a poor prognosis and inferior response to conventional treatment [7]. Erlotinib is a small molecule inhibitor that reversibly targets EGFR [8]. Several phase I/II trials have evaluated the feasibility of using erlotinib in combination with CRT based on cisplatin [9]. In the present study, we tested whether using erlotinib and paclitaxel instead of more toxic chemotherapeutic agents in combination with concurrent radiotherapy would improve treatment outcomes. This phase II study was designed to evaluate the safety and efficacy of paclitaxel 
Table 1: Baseline patient characteristics

\begin{tabular}{|c|c|c|}
\hline Characteristic & No. of patients & $\%$ \\
\hline All Patients & 21 & 100 \\
\hline \multicolumn{3}{|l|}{ Gender } \\
\hline $\begin{array}{l}\text { Male } \\
\text { Female }\end{array}$ & $\begin{array}{l}17 \\
4\end{array}$ & $\begin{array}{l}81 \\
19\end{array}$ \\
\hline \multicolumn{3}{|l|}{ Age (years) } \\
\hline $\begin{array}{l}\leq 60 \text { years } \\
60-70 \text { years } \\
>70 \text { years }\end{array}$ & \begin{tabular}{|l|}
10 \\
7 \\
4 \\
\end{tabular} & $\begin{array}{l}47 \\
33 \\
20 \\
\end{array}$ \\
\hline $\begin{array}{l}\text { ECOG PS } \\
0 \text { and } 1 \\
2\end{array}$ & $\begin{array}{l}20 \\
1\end{array}$ & \begin{tabular}{|l}
95 \\
5
\end{tabular} \\
\hline $\begin{array}{l}\text { TNM stage (UICC 2002) } \\
\text { II } \\
\text { IIIA } \\
\text { IIIB } \\
\text { IIIC }\end{array}$ & $\begin{array}{l}1 \\
13 \\
0 \\
7 \\
\end{array}$ & \begin{tabular}{|l|}
5 \\
62 \\
0 \\
33 \\
\end{tabular} \\
\hline $\begin{array}{l}\text { Location } \\
\text { Cervical } \\
\text { Upper } \\
\text { Middle } \\
\text { Lower } \\
\end{array}$ & \begin{tabular}{|l}
4 \\
10 \\
5 \\
2 \\
\end{tabular} & \\
\hline $\begin{array}{l}\text { EGFR mutation }(n=10) \\
\text { Positive } \\
\text { Negative }\end{array}$ & \begin{tabular}{|l|}
0 \\
10
\end{tabular} & \begin{tabular}{|l}
0 \\
100
\end{tabular} \\
\hline $\begin{array}{l}\text { EGFR expression } \\
\text { Positive } \\
\text { Negative }\end{array}$ & $\begin{array}{l}16 \\
5\end{array}$ & $\begin{array}{l}76 \\
24\end{array}$ \\
\hline $\begin{array}{l}\text { VEGF expression } \\
\text { Positive } \\
\text { Negative }\end{array}$ & \begin{tabular}{|l}
7 \\
14
\end{tabular} & \begin{tabular}{|l|}
34 \\
66
\end{tabular} \\
\hline $\begin{array}{l}\text { Smoking status } \\
\text { Smokers } \\
\text { Non-smokers }\end{array}$ & \begin{tabular}{|l}
7 \\
14
\end{tabular} & $\begin{array}{l}33 \\
67\end{array}$ \\
\hline
\end{tabular}

ECOG PS, Eastern Cooperative Oncology Group performance status; TNM, tumor-node-metastasis; EGFR, epidermal growth factor receptor; VEGF, vascular endothelial growth factor.

combined with erlotinib and concurrent radiotherapy for the treatment of inoperable ESCC.

\section{RESULTS}

\section{Patient characteristics}

Twenty-one patients were enrolled in the trial between October 2011 and October 2013. The median length of follow-up for patients who survived to the time of analysis was 21 months. The majority of the patients were male (17/21). The median age was 61 years (range, 40-77 years). The most common site of the primary tumor was the upper thoracic esophagus $(n=10)$, followed by the middle thoracic esophagus $(n=5)$, cervical esophagus $(n=4)$, and lower thoracic esophagus $(n=2)$. The majority of patients had stage III disease (20/21). Stage II, IIIA and IIIC primary disease was observed in 1, 13 and 7 patients, respectively. Preexisting cardiovascular disease, cerebrovascular disease, and diabetes were recorded in 3, 2 and 1 patients, respectively. Baseline patient and tumor characteristics are summarized in Table 1.

\section{Treatment toxicities}

The main treatment toxicities observed in our study are listed in Table 2 . The most common side effects were esophagitis in 18 patients (85\%), followed by hypoleukemia in 5 patients $(23.8 \%)$, fatigue in 4 patients $(19.0 \%)$, pulmonary toxicity in 3 patients $(14.2 \%)$, and skin rash in 2 patients $(9.5 \%)$. No patients developed diarrhea or nausea. Radiation pneumonitis was observed in 2 patients $(9.5 \%)$ : one patient developed grade 1 radiation pneumonitis, while the second developed grade 2 . The erlotinib dose had to be reduced in one patient. For the same patient, the radiation dose had to be decreased to 40 Gy due to grade 4 pulmonary toxicity (diffuse alveolar 

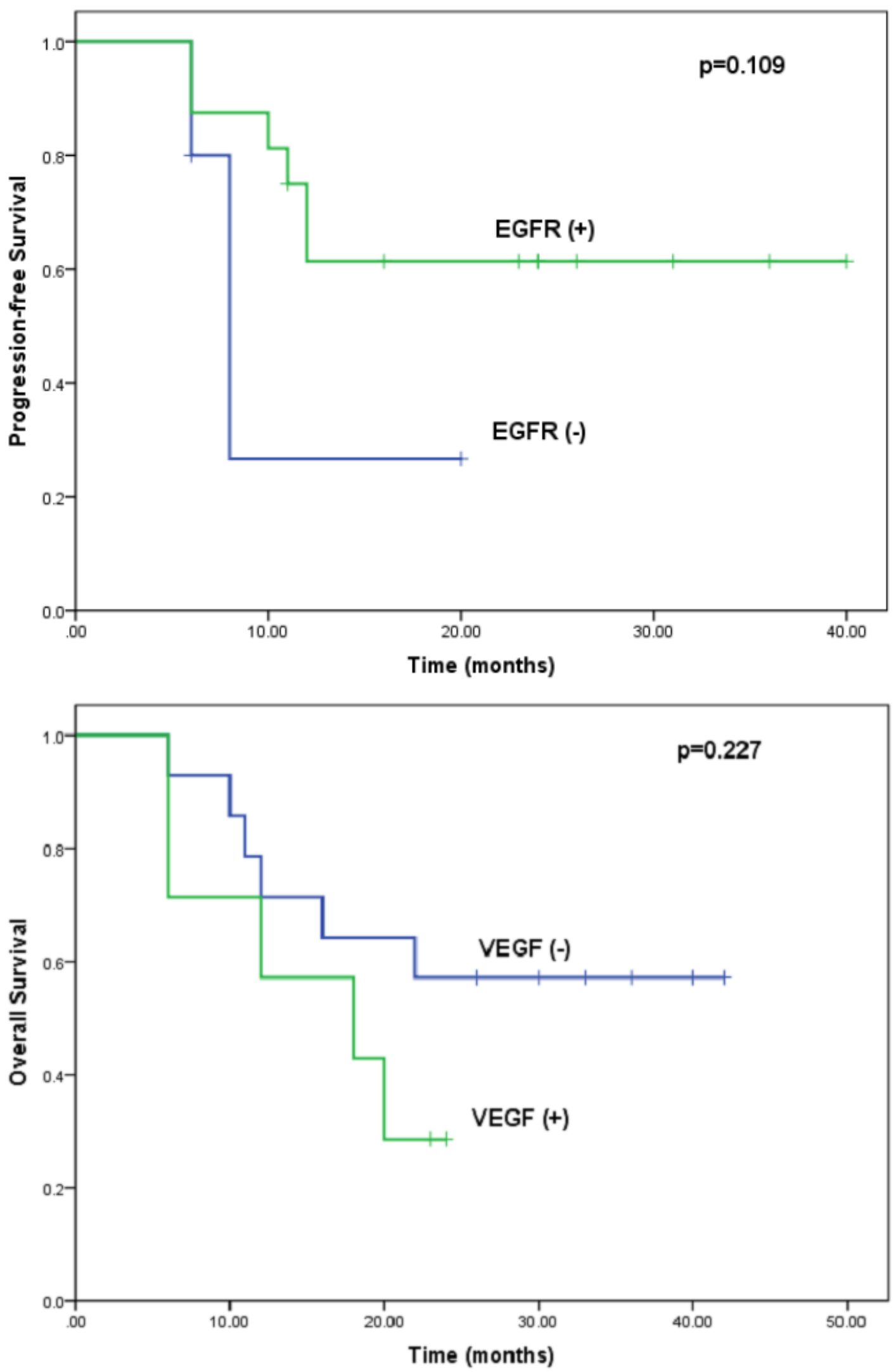

Figure 1: Kaplan-Meier curves comparing OS and PFS among patients with and without EGFR or VEGF expression. A. Kaplan-Meier curves for PFS stratified based on EGFR expression (log-rank test: $p=0.109)$. B. Kaplan-Meier curves for OS stratified based on VEGF expression (log-rank test: $p=0.227$ ). 
Table 2: Treatment-related toxicities

\begin{tabular}{|l|l|l|l|l|l|l|l|l|}
\hline & \multicolumn{3}{|l|}{$\begin{array}{l}\text { Grade 1 } \\
n \%\end{array}$} & \multicolumn{2}{l}{$\begin{array}{l}\text { Grade 2 } \\
n \%\end{array}$} & \multicolumn{2}{l|}{$\begin{array}{l}\text { Grade 3 } \\
n \%\end{array}$} & \multicolumn{3}{l|}{$\begin{array}{l}\text { Grade 4 } \\
n \%\end{array}$} \\
\hline Esophagitis & 7 & 34 & 9 & 52 & 2 & 9 & 0 & 0 \\
\hline Hypoleukemia & 2 & 10 & 3 & 14 & 1 & 5 & 0 & 0 \\
\hline Fatigue & 2 & 10 & 2 & 10 & 0 & 0 & 0 & 0 \\
\hline Pulmonary toxicities & 1 & 5 & 1 & 5 & 0 & 0 & 1 & 5 \\
\hline Skin rash & 1 & 5 & 1 & 5 & 0 & 0 & 0 & 0 \\
\hline Nausea & 0 & 0 & 0 & 0 & 0 & 0 & 0 & 0 \\
\hline Diarrhea & 0 & 0 & 0 & 0 & 0 & 0 & 0 & 0 \\
\hline Thrombocytopenia & 2 & 10 & 1 & 5 & 0 & 0 & 0 & 0 \\
\hline
\end{tabular}

hemorrhage), though the patient recovered after treatment [10]. Grade 3 hypoleukemia was observed in one patient $(4.7 \%)$. No additional toxicities greater than grade 3 and no treatment interruptions were recorded.

\section{Treatment outcomes}

Among the 21 patients treated, 8 (38.0\%) achieved a complete response (CR), $10(47.6 \%)$ a partial response (PR), and $3(14.4 \%)$ stable disease (SD). No patient showed progressive disease (PD). The median local progression-free survival (LPFS), progression-free survival (PFS), and overall survival (OS) were 17.5 months (95\% confidence interval [CI]: 13.9-24.0 months), 14.3 months (95\% CI: 12.4-21.9 months), and 22.9 month (95\% CI: 16.5-27.2 months), respectively. Two-year LPFS, PFS, and OS were $52.4 \%, 42.8 \%$, and $67.0 \%$, respectively.

\section{EGFR mutations and treatment outcomes}

Ten patient samples were evaluated for EGFR mutations; however, direct sequencing analysis revealed no mutations in any of the tumor samples, likely due to the low mutation rate reported in ESCC [11]. EGFR mutation analysis was therefore discontinued.

\section{EGFR/vascular endothelial growth factor (VEGF) expression patterns and treatment outcomes}

EGFR/VEGF expression was evaluated in patients from whom there were sufficient biopsy specimens for immunohistochemical examination. Twenty-one biopsy specimens were evaluated. Tumors from 5 patients exhibited no detectable EGFR expression, whereas those from the remaining 16 patients showed EGFR expression. Tumors from 7 patients showed VEGF expression, while those from the remaining 14 patients had no detectable VEGF expression. Patients with tumors in which EGFR expression was detected tended to have longer PFS, while those with tumors expressing VEGF tended to have worse outcomes, but these differences were not statistically significant ( $p>0.05$; Figure 1A, 1B).

\section{Smoking status and treatment outcomes}

Of the 21 patients treated, 7 had never smoked. The PFS was significantly better among these patients than among those who smoked (18.1 vs. 11.5 months, $p<0.05$; Figure 2A), as was OS (24.3 vs. 13.7 months, $p>0.05$; Figure 2B).

\section{DISCUSSION}

For patients with unresectable, locally advanced ESCC, the prognosis is poor, despite the standard care of concurrent chemoradiotherapy [12]. In an earlier study (RTOG-8501), the 2-year survival rate among ESCC patients was only $36 \%$ [13]. In this palliative setting, the efficacy of aggressive combination chemotherapy has been questioned. First, the combination chemotherapy regimen provided effective palliation but resulted in substantial toxicity, with only $59 \%$ of patients completing the planned chemoradiation. Second, perhaps due to the high level of toxicity associated with the chemotherapy regimen, the total radiation dose was only 50 Gy in this trial, which is widely considered insufficient for radical treatment of ESCC patients. Similar results were obtained in several 

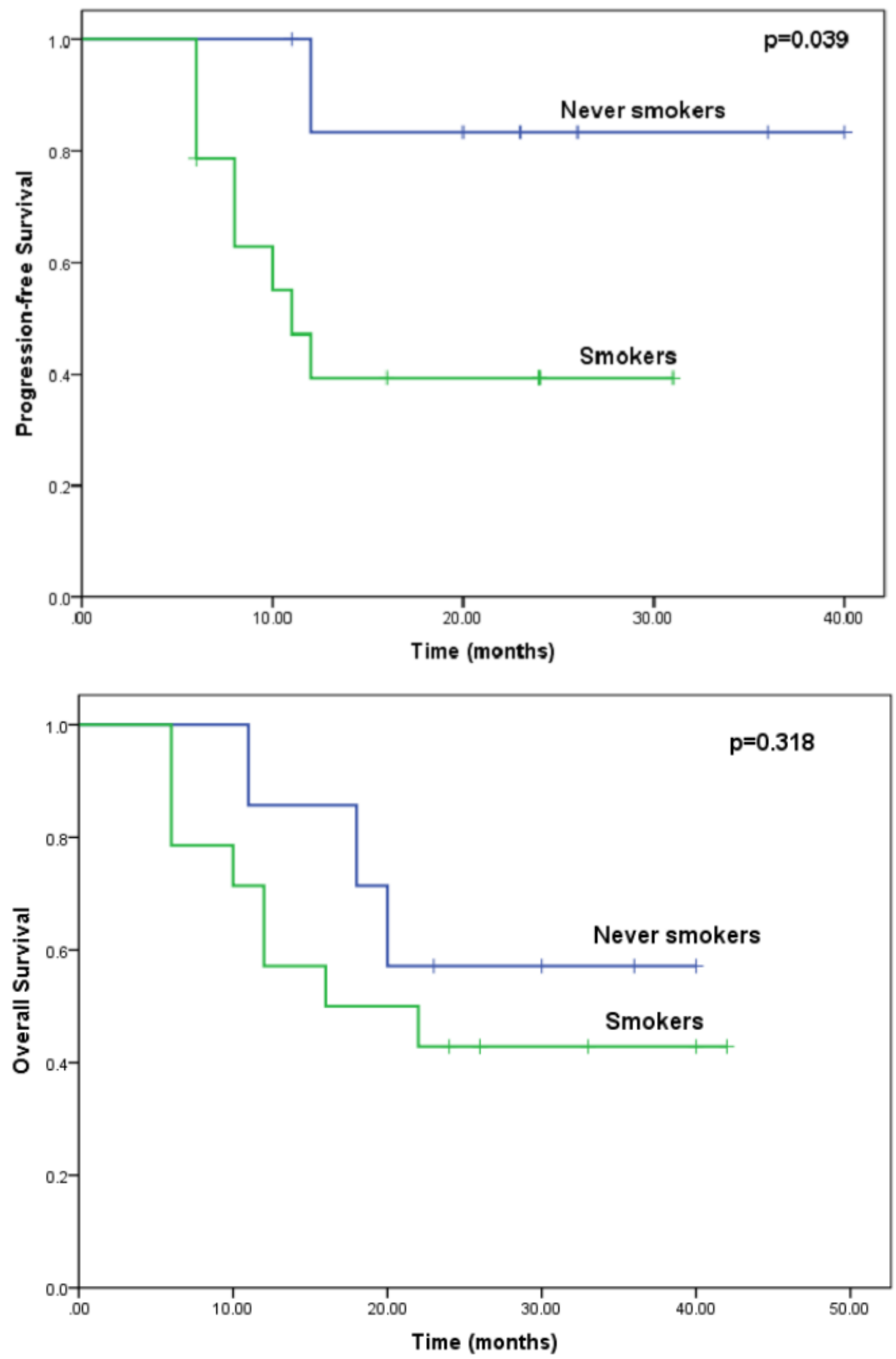

Figure 2: Kaplan-Meier curves comparing OS and PFS among patients with and without a history of smoking. A. Kaplan-Meier curves for PFS stratified based on tobacco smoking status (log-rank test: $p=0.039$ ). B. Kaplan-Meier curves for OS stratified based on tobacco smoking status (log-rank test: $p=0.318$ ). 
other small-sample studies: the response rate, including CR and PR, was approximately $60-70 \%$, while the median survival time was 10-12 months; 2-year local failure and OS were $25-60 \%$ and $20-40 \%$, respectively [14]. These results highlight the need to explore new less toxic agents that could potentially replace combined chemotherapy to improve outcomes.

EGFR overexpression correlates with poor prognosis and an inferior response to therapy [18]. This affords a potential opportunity for anti-EGFR agents to improve treatment outcomes. Moreover, a strong rationale may exist for combining erlotinib, an EGFR inhibitor, with RT. First, erlotinib disrupts cell growth pathways and enhances the sensitivity of cells to RT [19]. Second, by exerting a cytoreductive effect and creating a hypoxic environment, RT may enhance the effectiveness of erlotinib [20]. Finally, the toxicities associated with erlotinib, RT and paclitaxel do not overlap, which enables their concomitant use. Hence, it is reasonable to use erlotinib concurrently with chemoradiation in ESCC patients.

In a phase I study to evaluate the combination of erlotinib with cisplatin-based CRT, ESCC patients were assigned to receive a dose of 50,100 , or $150 \mathrm{mg} /$ day erlotinib concurrently with RT and cisplatin or PF [21]. Erlotinib was well tolerated at $150 \mathrm{mg}$ /day. A phase II study found that RT with concurrent PF and erlotinib for patients with locally advanced ESCC had antitumor activity, but was accompanied by significant toxicities. Acute grade 3 toxicities included leukopenia, esophagitis, and skin rash; 4 of 22 patients were unable to complete both cycles of chemotherapy [22]. These initial results suggest that erlotinib has the potential to improve the outcomes of ESCC patients when combined with a more reasonable chemotherapy scheme.

In China, paclitaxel is widely used instead of PF in CRT for esophageal cancer. This regimen reduces toxicities and enables the RT dose to be increased [15]. Traditionally, paclitaxel is administered at $175-225 \mathrm{mg} / \mathrm{m}$ ${ }^{2}$ over 3 h every 3 weeks. A Cochrane review examining various schedules of paclitaxel infusion in patients with advanced adenocarcinoma concluded that lower doses at more frequent intervals were more effective [16]. Studies comparing metronomic weekly paclitaxel to multidrug combination chemotherapy found that the metronomic scheduling achieved similar clinical benefits with less toxicity [17].

To the best of our knowledge, this is the first study to evaluate the safety and efficacy of erlotinib and paclitaxel combined with RT for ESCC. Concomitant treatment with erlotinib, paclitaxel and RT in locally advanced ESCC patients ineligible for surgery had significant clinical value, and the toxicities were tolerable. The overall response rate $(\mathrm{CR}+\mathrm{PR})$ was $85.6 \%$, and the treatment yielded satisfactory 2 -year OS and localregional control. Furthermore, the present study is the first to correlate EGFR and VEGF expression with treatment outcomes. Immunohistochemical examination confirmed that tumors from 16 patients $(76 \%)$ expressed EGFR. This is consistent with the earlier observation that EGFR is overexpressed in 30-90\% of ESCC cases [23]. Our results also indicate that patients with ESCC expressing EGFR had a better PFS than patients with no EGFR expression. By contrast, patients expressing VEGF had a worse OS, though the difference was not significant, perhaps due to the small sample size.

Studies of non-small cell lung cancer (NSCLC) showed that patients with tumors expressing EGFR carrying a mutation were more likely to respond to erlotinib [24]. However, the mechanism of the combined erlotinib and RT differs from erlotinib alone in NSCLC. In vitro studies indicate that erlotinib enhances the radiation response at several levels, including cell cycle arrest, apoptosis induction, accelerated cellular repopulation, and DNA damage repair [25]. Erlotinib modulates the radiation response by influencing cell cycle kinetics and apoptosis. Erlotinib in combination with RT reduces the number of cells in S phase while increasing the level of apoptosis and promoting an increase in sensitivity to RT [19]. In vivo, erlotinib influences the expression of radiation response genes from several functional classes, including those involved in cell cycle arrest and DNA damage repair [26]. In a preclinical study involving three human cancer cell lines with low, moderate, or very high EGFR expression, the extent of erlotinib-induced radiosensitization was proportional to the EGFR expression level [27]. The cell line expressing very high levels of EGFR exhibited the strongest radioresistance, and treatment with erlotinib increased the extent of G1 arrest and augmented apoptosis in those cells. In addition, the anti-angiogenic agent bevacizumab in combination with erlotinib and RT significantly decreased growth of human VEGF-secreting cell xenografts [26]. These results from preclinical studies highlight the potential benefit of both EGFR and VEGF inhibition.

We found that non-smokers had a better treatment response to erlotinib combined with CRT than smokers, which was also seen in NSCLC patients [28]. Other studies have shown that tobacco smoking can lower the response to erlotinib by reducing its bioavailability by $25 \%$ and increasing its clearance rate [29].

Tissues such as skin and gut express EGFR constitutively, which may explain the toxicity associated with erlotinib. In NSCLC studies, skin rash and diarrhea, the major side effects reported, occurred in $47-86 \%$ and $30-70 \%$ of patients, respectively; however, they were mostly grade $1 / 2$ [30]. In our study, only two patients had grade I skin rashes, and no patients developed diarrhea. Despite the small sample size limiting our ability to draw a definite conclusion, it is noteworthy that erlotinib may have different toxicities in different tumor types, which is consistent with other studies [23].

The present study has several limitations. First, 
although the results are exciting, this is a single arm, small sample, phase II study. Large, randomized trials will be needed to confirm the benefits of erlotinib administered concurrently with CRT. Second, other molecular markers in addition to EGFR and VEGF should also be identified to predict patient populations that will benefit from erlotinib combined with CRT in ESCC. Third, erlotinib was administered concurrently with radiation; whether other dosing schedules could improve outcomes needs further study.

In conclusion, our data suggest that weekly paclitaxel combined with erlotinib and concurrent radiotherapy shows promise as an effective, tolerated regimen for patients with inoperable ESCC. Our study revealed better outcomes in patients with EGFR expression, no VEGF expression, and no history of smoking. A future randomized study is needed to confirm the benefits of concomitant treatment with erlotinib and chemoradiotherapy in ESCC patients. To predict the TKI response, valuable molecular biomarkers or clinical factors should be exploited in the future.

\section{MATERIALS AND METHODS}

\section{Study design}

We performed an open-label, single arm, phase II trial with patients recruited from our Department of GI Oncology. Patients received CRT with erlotinib (150 $\mathrm{mg}$ /day). CRT consisted of paclitaxel $45 \mathrm{mg} / \mathrm{m}^{2}$ weekly concurrently with 60 Gy of radiotherapy given in 30 fractions. The primary endpoint was 2-year OS and PFS. LPFS and toxicities were secondary endpoints. This trial was registered on the Clinical Trials website, reference number NCT 01752205.

\section{Patient population}

Inclusion criteria consisted of histologically confirmed squamous cell carcinoma of the esophagus or esophagogastric junction; ineligibility for surgery; Eastern Cooperative Oncology Group (ECOG) performance status (PS) score $0-2$; age $\geq 18$ years old; life expectancy $\geq 12$ weeks; no prior palliative therapy; at least one bidimensional measurable disease as defined by RECIST ver 1.1; adequate organ function for treatment; absolute neutrophil count $(\mathrm{ANC}) \geq 1000$ cells $/ \mathrm{mm}^{3}$; platelets $\geq 100000$ cells $/ \mathrm{mm}^{3}$; estimated creatinine clearance $\geq 50$ $\mathrm{mL} / \mathrm{min}$, or serum creatinine $<1.5 \times$ institutional upper limit of normal (ULN); bilirubin $\leq 1.5 \times$ ULN; AST $($ SGOT) $\leq 2.5 \times$ ULN $(5.0 \times$ ULN if hepatic metastases); ALT $(\mathrm{SGPT}) \leq 2.5 \times$ ULN $(5.0 \times$ ULN if hepatic metastases $)$; 12-lead electrocardiogram (ECG) with normal tracing or non-clinically significant changes that do not require medical intervention; QTc interval $\leq 470 \mathrm{~ms}$ and without history of Torsades de Pointes or other symptomatic QTc abnormality; LVEF (by MUGA or echocardiogram) of $\geq 50 \%$. Exclusion criteria included prior thoracic radiotherapy or anti-EGFR treatment, uncontrolled brain metastasis, and other malignancies. All patients provided signed informed consent before participating in this study and were amenable to compliance with protocol schedules. The study was approved by the institutional ethics committee of the 307 Hospital of PLA.

Pre-enrollment examinations included physical examination, full blood counts, biochemistry tests, electrocardiograph, thoracic computed tomography (CT) and/or 18F-fluorodeoxy glucose positron emission tomography (18F-FDG PET), barium esophagram, cervical and abdominal ultrasound examination, endoscopic ultrasonography and bone scan. Disease was staged according to the 7 th edition of the American Joint Committee on Cancer Staging System. During treatment, physical examination and full blood counts were repeated weekly, and biochemistry tests were repeated every two weeks.

\section{Treatment procedure}

The treatment plan included administration of erlotinib (150 mg daily) for a period of 60 days beginning at the start of radiotherapy, and paclitaxel weekly along with intensity modulated conformal radiation therapy (IMRT). Gross tumor volume (GTV) was determined based on thoracic CT (or 18F-FDG PET if possible) and the endoscopy results. Clinical target volume (CTV) included the GTV and supraclavicular and mediastinal lymph node regions. The celiac lymph node region was also included in the CTV for tumors located in the lower third of the esophagus. Planning target volumes (PTV) were generated as the CTV plus $5 \mathrm{~mm}$ of margin. Planning objectives involved delivering $60 \mathrm{~Gy}$ in 30 fractions of 2 Gy per fraction over 6 weeks to obtain 95\% PTV coverage. Dose modification for hematological toxicity was based on full blood counts taken within the 3 days before the start of weekly paclitaxel. Paclitaxel was omitted for grade 4 toxicity, and was reduced to $75 \%$ and $50 \%$ of the starting dose after the first and second occurrences of grade 3 toxicity, respectively. Sequential dose reduction of erlotinib to $100 \mathrm{mg} / \mathrm{d}$ was advised for second occurrences of grade 3 skin rash, and it was permanently discontinued after a third appearance.

\section{Immunohistochemical analysis}

Levels of EGFR and VEGF expression were assessed immunohistochemically. All tissue samples were immersion fixed in 4\% paraformaldehyde, embedded in paraffin, and sectioned at a thickness of $8 \mu \mathrm{m}$. Sections 
were probed first with anti-EGFR (1:100, rat polyclonal; Santa Cruz) or anti-VEGF (1:50, mouse monoclonal; Abcam) antibody overnight at $4^{\circ} \mathrm{C}$, and then with secondary antibodies for $1 \mathrm{~h}$ at room temperature.

\section{EGFR mutation analysis}

EGFR mutation was assessed using polymerase chain reaction (PCR)-direct sequencing. For gene mutation analysis, DNA was collected from primary esophageal tumor specimens using the phenol-chloroform extraction method after overnight digestion of the tissue using proteinase $\mathrm{K}$. Mutation within the four tyrosine kinase domain exons (18-21) of EGFR that are frequently mutated in esophageal cancer was assessed using PCRdirect sequencing as previously reported [11]. All sequence variants were confirmed through independent PCR amplification and sequencing in both directions.

\section{Statistical analysis}

In the only randomized trial designed to deliver adequate doses of PF with concurrent RT for ESCC (RTOG-8501), the two-year survival rate was $36 \%$. We anticipated a survival rate of $46 \%$ in our study. The statistical design was intended to enable us to detect a response rate of at least $20 \%$. It was calculated that a sample size of 20 patients was required.

Kaplan-Meier survival curves were estimated, and the log-rank test was applied to assess the differences in survival distributions related to EGFR expression or mutation and clinical variables. Statistical analysis was performed using SPSS software, version 20.0. All probability values were two-sided, and values of $p<0.05$ were considered statistically significant.

\section{CONFLICTS OF INTEREST}

The authors declare that there are no conflicts of interest.

\section{REFERENCES}

1. Mariette C, Piessen G, Triboulet JP. Therapeutic strategies in oesophageal carcinoma: role of surgery and other modalities. Lancet Oncol. 2007; 8: 545-553.

2. Pennathur A, Gibson MK, Jobe BA, Luketich JD. Oesophageal carcinoma. Lancet. 2013; 381: 400-412.

3. Cohen DJ, Leichman L. Controversies in the treatment of local and locally advanced gastric and esophageal cancers. J Clin Oncol. 2015; 33:1754-1759.

4. Lloyd S, Chang BW. Current strategies in chemoradiation for esophageal cancer. J Gastrointest Oncol. 2014; 5:156165.
5. Weiner LM. Paclitaxel in the treatment of esophageal cancer. Semin Oncol. 1999; 26:106-108.

6. Jiang Yong, Cui Lin, Wu Xingjun, He Xuejun, Liu Xingxiang, Zhou Xiangrong, Liu Jianjun. Radiosensitizing Effect of Weekly-dose Paclitaxel on Locally Advanced Esophageal Carcinoma.Zhong Liu Ji Chu Yu Lin Chuang. 2012; 6:489-491.

7. Pande AU, Iyer RV, Rani A, Maddipatla S, Yang GY, Nwogu CE, Black JD, Levea CM, Javle MM. Epidermal growth factor receptor-directed therapy in esophageal cancer. Oncology. 2007; 73:281-289.

8. Sutter AP, Höpfner M, Huether A, Maaser K, Scherübl H. Targeting the epidermal growth factor receptor by erlotinib (Tarceva) for the treatment of esophageal cancer. Int $\mathrm{J}$ Cancer. 2006; 118:1814-1822.

9. Mehta VK. Radiotherapy and erlotinib combined: review of the preclinical and clinical evidence. Front Oncol. 2012; $10 ; 2: 31$.

10. Zhao CH, Liu RR, Lin L, Liu JZ, Ge FJ, Li SS, Ye CY, Chen YL, Wang Y, Xu JM. Diffuse alveolar hemorrhage after erlotinib combined with concurrent chemoradiotherapy in a patient with esophageal carcinoma. Int J Clin Exp Med. 2014;7:4492-4497.

11. Cui Y, Chang D, Liu M, Lin C, Zhao B, Zhang X, Gong M. Identification of exon 19 and 21 mutations of EGFR gene in Chinese patients with esophageal squamous cell carcinoma. World J Surg Oncol. 2013; 9;11:266.

12. Shridhar R, Imani-Shikhabadi R, Davis B, Streeter OA, Thomas CR Jr. Curative treatment of esophageal cancer; an evidenced based review. J Gastrointest Cancer. 2013;44:375-384.

13. Cooper JS, Guo MD, Herskovic A, Macdonald JS, Martenson JA Jr, Al-Sarraf M, Byhardt R, Russell AH, Beitler JJ, Spencer S, Asbell SO, Graham MV, Leichman LL. Chemoradiotherapy of locally advanced esophageal cancer: long-term follow-up of a prospective randomized trial (RTOG 85-01). Radiation Therapy Oncology Group. JAMA. 1999;281:1623-1627.

14. D'Journo XB, Thomas PA. Current management of esophageal cancer. J Thorac Dis. 2014; 6 Suppl 2: S253-64.

15. Constantinou M, Tsai JY, Safran H. Paclitaxel and concurrent radiation in upper gastrointestinal cancers. Cancer Invest. 2003;21:887-896.

16. Williams C,Bryant A. Short versus long duration infusions of paclitaxel for any advanced adenocarcinoma. Cochrane Database Syst Rev. 2011;5:CD003911.

17. IIson DH,Wadleigh RG,Leichman LP,Kelsen DP.Paclitaxel given by a weekly 1-h infusion in advanced esophageal cancer. Ann Oncol. 2007;18:898-902.

18. Yewale C, Baradia D, Vhora I, Patil S, Misra A. Epidermal growth factor receptor targeting in cancer: a review of trends and strategies. Biomaterials. 2013;34:8690-8707.

19. Chinnaiyan P, Huang S, Vallabhaneni G, Armstrong E, Varambally S, Tomlins SA, Chinnaiyan AM, Harari PM. 
Mechanisms of enhanced radiation response following epidermal growth factor receptor signaling inhibition by erlotinib (Tarceva). Cancer Res. 2005;65:3328-3335.

20. Tortora G, Gelardi T, Ciardiello F, Bianco R. The rationale for the combination of selective EGFR inhibitors with cytotoxic drugs and radiotherapy. Cancer Res. 2007;22: S47-52.

21. Dobelbower MC, Russo SM, Raisch KP, Seay LL, Clemons LK, Suter S, Posey J, Bonner JA. Epidermal growth factor receptor tyrosine kinase inhibitor, erlotinib, and concurrent 5-fluorouracil, cisplatin and radiotherapy for patients with esophageal cancer: a phase I study. Anticancer Drugs. 2006 Jan;17(1):95-102.

22. Li G, Hu W, Wang J, Deng X, Zhang P, Zhang X, Xie $\mathrm{C}, \mathrm{Wu}$ S. Phase II study of concurrent chemoradiation in combination with erlotinib for locally advanced esophageal carcinoma. Int J Radiat Oncol Biol Phys. 2010 ;78:14071412.

23. Xu Y, Zheng Y, Sun X, Yu X, Gu J, Wu W, Zhang G, Hu J, Sun W, Mao W. Concurrent radiotherapy with gefitinib in elderly patients with esophageal squamous cell carcinoma: Preliminary results of a phase II study. Oncotarget. 2015 ;6:38429-38439. doi: 10.18632/oncotarget.5193.

24. Russo A, Franchina T, Ricciardi GR, Picone A, Ferraro G, Zanghì M, Toscano G, Giordano A, Adamo V. A decade of EGFR inhibition in EGFR-mutated non small cell lung cancer (NSCLC): Old successes and future perspectives. Oncotarget. 2015 ;6:26814-26825. doi: 10.18632/ oncotarget. 4254 .
25. Nyati MK, Morgan MA, Feng FY, Lawrence TS. Integration of EGFR inhibitors with radiochemotherapy. Nat Rev Cancer. 2006 ;6:876-885.

26. Bozec, A, Sudaka, A, Fischel, J. L, Brunstein, M. C, Etienne-Grimaldi, M. C, Milano G. Combined effects of bevacizumab with erlotinib and irradiation: a preclinical study on a head and neck cancerorthotopic model. Br J Cancer. 2008 ;99:93-99.

27. Kim KS, Jeong JY, Kim YC, Na KJ, Kim YH, Ahn SJ, Baek SM, Park CS, Park CM, Kim YI, Lim SC, Park KO. Predictors of the response to gefitinib in refractory nonsmall cell lung cancer. Clin Cancer Res. 2005;11:22442251.

28. Sim SH, Han SW, Oh DY, Lee SH, Kim DW, Im SA, Chung DH, Kim TY, Lee JS, Kim YW, Heo DS, Bang YJ. Erlotinib after Gefitinib failure in female never-smoker Asian patients with pulmonary adenocarcinoma.Lung Cancer. 2009;65:204-207.

29. Li X,KameneckaTM,Cameron MD.Bioactivation of the epidermal growth factor receptor inhibitor gefitinib:implications for pulmonary and hepatic toxicities. Chem Res Toxicol.2009;22:1736-1742.

30. Liu HB, Wu Y, Lv TF, Yao YW, Xiao YY, Yuan DM, Song Y. Skin rash could predict the response to EGFR tyrosine kinase inhibitor and the prognosis for patients with non-small cell lung cancer: a systematic review and metaanalysis. PLoS One. 2013;8:e55128. 